\title{
EDITORIAL
}

\section{Iron chelation therapy in myelodysplastic syndrome - Cui bono?}

\author{
Leukemia (2009) 23, 1373; doi:10.1038/leu.2009.39
}

One of the earlier names used to describe myelodysplastic syndromes (MDS) was 'refractory anemia' because of their resistance to the therapy known at the time. ${ }^{1}$ It is ironic that 'refractory anemia' of yesteryear remains difficult to treat as we enter the 21 st century, although notable progress has been made in terms of allogeneic stem cell transplantation. ${ }^{2}$ Currently available drugs for MDS are not effective in eradicating the abnormal disease clone but can sometimes induce transient remissions. Most noteworthy in this regard are lenalidomide, which is particularly effective in the presence of $\operatorname{del}(5 q){ }^{3}$ and hypo-methylating agents, which are capable of slowing down blastic transformation ${ }^{4,5}$ or even perhaps prolong survival by 9 months. $^{4}$

So what about iron chelation therapy for MDS? On 2 November 2005, the Food and Drug Administration approved deferasirox (Exjade, Novartis, East Hanover, NJ, USA), an oral iron-chelating agent, for use in transfusional hemosiderosis associated with hemoglobinopathies, rare anemias and MDS. This was welcome news at least in thalassemia, a disease in which iron chelation therapy improves survival in transfusiondependent patients. However, the same is not necessarily true for patients with MDS. This should come as no surprise because complications of iron overload develop after many years of target organ exposure; more than $85 \%$ of MDS patients are diagnosed after age 60 years and 3-year survival is only $35 \%{ }^{5}$

Is there a subset of MDS patients that would benefit from iron chelation therapy? A life expectancy of over 5 years is possible in MDS patients with $<5 \%$ bone marrow blasts and absence of both multilineage dysplasia and high-risk cytogenetic abnormalities. ${ }^{6}$ However, what is often overlooked in this setting is the fact that the transfusional need in such patients is associated with inferior overall and leukemia-free survival, and therefore heralds premature death from progressive disease and not necessarily from transfusional hemosiderosis. ${ }^{6,7}$ Furthermore, increased serum ferritin, which is often used as a surrogate for iron overload, strongly correlates with transfusion load, making it difficult to decipher its independent prognostic value. Even if increased serum ferritin were independently associated with shortened survival, it could be equally ascribed to its property as an acute-phase reactant as it would as a surrogate for iron overload.

Increased serum ferritin in transfusion-dependent cancer patients is a fact and not necessarily a problem. Myocardial or hepatic iron deposition is seldom cited as a cause of death or morbidity in MDS; as for the few anecdotal cases, it is uncertain that they would have fared better had they received one of the currently available iron-chelating agents. With regard to other cardiac events, it is disingenuous to imply a contributory role for iron overload in the absence of controlled evidence that takes into account other risk factors including anemia. When deferoxamine, which has to be given subcutaneously over a long period of the day, was the only available iron chelator, few doctors or patients would accept this therapy. The mere fact that a chelator has an easier route of administration does not justify its use in MDS given the associated toxicity and expense plus unknown clinical benefit. Controlled clinical trials, and not a litany of consensus statements, are needed to justify such treatment. Primary outcome measures in such trials must portray meaningful health outcome rather than a banal effect on laboratory surrogates of iron overload.

$$
\begin{array}{r}
\text { A Tefferi }{ }^{1} \text { and RM Stone }{ }^{2} \\
{ }^{1} \text { Mayo Clinic, Rochester, MN, USA and } \\
{ }^{2} \text { Dana-Farber Cancer Institute, Harvard Medical School, } \\
\text { Boston, MA, USA } \\
\text { E-mail: tefferi.ayalew@mayo.edu }
\end{array}
$$

\section{References}

1 Bomford RR, Rhodes CP. Refractory anemia. Q J Med 1941; 10: 175-281.

2 Chang C, Storer BE, Scott BL, Bryant EM, Shulman HM, Flowers ME et al. Hematopoietic cell transplantation in patients with myelodysplastic syndrome or acute myeloid leukemia arising from myelodysplastic syndrome: similar outcomes in patients with de novo disease and disease following prior therapy or antecedent hematologic disorders. Blood 2007; 110: 1379-1387.

3 List A, Dewald G, Bennett J, Giagounidis A, Raza A, Feldman E et al. Lenalidomide in the myelodysplastic syndrome with chromosome 5q deletion. N Engl J Med 2006; 355: 1456-1465.

4 Fenaux P, Mufti GJ, Santini V, Finelli C, Giagounidis A, Schoch R et al. Azacitidine (AZA) treatment prolongs overall survival (OS) in higher-risk MDS patients compared with conventional care regimens (CCR): results of the AZA-001 Phase III Study. ASH Annual Meeting Abstracts 2007; 110: 817.

5 Ma X, Does M, Raza A, Mayne ST. Myelodysplastic syndromes: incidence and survival in the United States. Cancer 2007; 109: 1536-1542.

6 Malcovati L, Porta MG, Pascutto C, Invernizzi R, Boni M, Travaglino E et al. Prognostic factors and life expectancy in myelodysplastic syndromes classified according to WHO criteria: a basis for clinical decision making. J Clin Oncol 2005; 23: 7594-7603.

7 Chee CE, Steensma DP, Wu W, Hanson CA, Tefferi A. Neither serum ferritin nor the number of red blood cell transfusions affect overall survival in refractory anemia with ringed sideroblasts. Am J Hematol 2008; 83: 611-613. 